\title{
Cell cycle-related kinase is a crucial regulator for ciliogenesis and Hedgehog signaling in embryonic mouse lung development
}

\author{
Hankyu Lee ${ }^{1} \&$ Hyuk Wan Ko ${ }^{2, *}$ \\ ${ }^{1}$ College of Pharmacy, Dongguk University-Seoul, Goyang 10326, ${ }^{2}$ Department of Biochemistry, College of Life Science and Biotechnology, \\ Yonsei University, Seoul 03722, Korea
}

Cell cycle-related kinase (CCRK) has a conserved role in ciliogenesis, and Ccrk defects in mice lead to developmental defects, including exencephaly, preaxial polydactyly, skeletal abnormalities, retinal degeneration, and polycystic kidney. Here, we found that Ccrk is highly expressed in mouse trachea and bronchioles. Ccrk mutants exhibited pulmonary hypoplasia and abnormal branching morphogenesis in respiratory organ development. Furthermore, we demonstrated that Ccrk mutant lungs exhibit not only impaired branching morphogenesis but also a significant sacculation deficiency in alveoli associated with reduced epithelial progenitor cell proliferation. In pseudoglandular stages, Ccrk mutant lungs showed a downregulation of Hedgehog $(\mathrm{Hh})$ signaling and defects in cilia morphology and frequency during progenitor-cell proliferation. Interestingly, we observed that activation of the Hh signaling pathway by small-molecule smoothened agonist (SAG) partially rescued bud morphology during branch bifurcation in explants from Ccrk mutant lungs. Therefore, CCRK properly regulates respiratory airway architecture in part through $\mathrm{Hh}$-signal transduction and ciliogenesis. [BMB Reports 2020; 53(7): 367-372]

\section{INTRODUCTION}

The architecture of the mammalian respiratory system is characterized by a tree-like structure composed of proximal air-conducting tubes and a surface for distal gas exchange (1). The emergence of two primary lung buds from the ventral side of the anterior foregut follows and corresponds to the embryonic stage of lung development (E9.5-12.5). Subsequently, during the pseudoglandular stage (E12.5-16.5) a bronchial tree grows via continuous formation of new distal branch buds (2). Proliferative expansion of endodermal progenitors at these sites requires

*Corresponding author. Tel: +82-2-2123-2699; Fax: +82-2-362-9897; E-mail: kohw@yonsei.ac.kr

https://doi.org/10.5483/BMBRep.2020.53.7.295

Received 28 November 2019, Revised 10 December 2019, Accepted 16 December 2019

Keywords: Branching morphogenesis, Cell cycle-related kinase, Hedgehog signaling, Lung development, Primary cilia signals from the adjacent mesenchyme (3). Respiratory system development involves a diverse process of cell proliferation and differentiation, precisely regulated by interactions between various signaling molecules and regulation of target-gene expression by transcription factors $(2,3)$.

Hedgehog $(\mathrm{Hh})$ signal transduction is a key regulator of cell differentiation, proliferation, and cell cycle progression during the developmental processes of many organs in embryo. Disruption of $\mathrm{Hh}$ signaling leads to prenatal lethality, with abnormal development of the neural tube, eye, and gastrointestinal tract in mice (4). Hh ligands bind to the transmembrane receptor Patched (Ptch1) to derepress the $\mathrm{G}$ protein-coupled receptor (GPCR)-like membrane protein Smoothened (Smo). Activated Smo concentrates on the tip of primary cilia to activate the Gli family transcription factors, albeit through unknown mechanisms.

In addition, Sonic hedgehog (SHH), a mammalian counterpart of $\mathrm{HH}$, is produced from lung endodermal epithelial cells during lung development and affects developing mesenchymal cells to regulate the proliferation, differentiation, and patterning of both the epithelium and the mesenchyme $(5,6)$. Consistently, evidence from Shh mutant mice showed tracheoesophageal abnormalities, including hypoplastic lungs, caused by defects in branching morphogenesis (5). Moreover, Gli2 or Gli3 mutant mice display different types of respiratory system abnormalities that are similar to those defects observed in Shh mutant mice $(7,8)$. Therefore, the Hh-signaling pathway is important for respiratory system development, requiring tight spatial and temporal regulation to ensure its correct function.

The Hh signaling pathway is tightly linked to the primary cilium, which functions as a signaling center. Most ciliary gene mutant mice exhibit structural or functional malformation of cilia and defective embryo development caused by dysregulation of important development signaling pathways (9). Moreover, defective primary cilia are associated with diverse human congenital genetic diseases, termed ciliopathies (10). Recent studies of the early developmental stages of the lung revealed the presence of primary cilia for a short time in non-proliferating tracheal epithelial cells (11). Primary cilia are transiently present during airway epithelial cell differentiation and give way to motile cilia at later stages of development (11); however, the role of primary cilia in the early development of the respiratory system remains unresolved. 
CCRK, known as cyclin-dependent kinase 20 (CDK20), regulates primary cilia integrity and the $\mathrm{Hh}$ signaling pathway in neural patterning during embryo development. Moreover, multiple developmental organogenesis defects were observed in $C c r k$ knockout $\left(\mathrm{Ccrk}^{\mathrm{KO}}\right)$ embryos and are associated with dysregulation of $\mathrm{Hh}$ signaling and defects in ciliary structure and distribution (12). CCRK is the homolog of Chlamydomonas long flagella 2 (LF2), which is composed of a long flagella complex (LRC) and is involved in the assembly of the flagella. Consistently, morpholino-mediated knockdown of either Ccrk or broad-minded (Bromi), a CCRK-interacting protein, in zebrafish results in nearly identical phenotypes in ciliary shape and affected tissues, suggesting that their functions are closely linked to the architecture of the primary cilium (13). Here, we demonstrated that loss of Ccrk leads to defective branching morphogenesis and hypoplasia because of decreased epithelial cell proliferation. Downregulation of Hh signaling is accompanied by abnormal ciliogenesis in Ccrk mutant lungs. Interestingly, pharmacological activation of Hh signaling partially rescued branching morphogenesis in Ccrk mutant lungs. Collectively, these findings suggest that Ccrk may play a key role in lung development via $\mathrm{Hh}$ signaling and ciliogenesis, because loss of Ccrk leads to defective branching and pulmonary hypoplasia in vivo.

\section{RESULTS}

Loss of Ccrk leads to hypoplastic lung development with defective branching morphogenesis

Previously, our group reported that $\mathrm{CCrk}^{\mathrm{KO}}$ mice display developmental defects in multiple organs that resulted from dysregulation of the primary cilia and Hh signaling in mouse embryos (12). Using whole-mount lacZ staining on heterozygote knockout-first conditional Ccrk embryos (Ccrk ${ }^{\text {tm1a/t }}$ ) at E15.5, we observed higher CCRK expression in embryonic trachea and pulmonary bronchioles (Supplementary Fig. 1). To uncover the role of CCRK in the pulmonary system, we phenotypically analyzed lung development in previously generated Ccrk null mutant embryos $\left(\mathrm{Ccrk}^{\mathrm{KO}}\right)$ or control $\left(\mathrm{Ccrk} \mathrm{K}^{W T}\right)$ littermates (12). Deletion of the Ccrk gene causes hypoplastic lung morphology around E14.5 (Fig. 1A). The lungs of $\mathrm{CCrk}^{\mathrm{KO}}$ mutant embryos were smaller and had fewer lobes than the control had. To find the cause of the underdeveloped lung in $\mathrm{Ccrk}^{\mathrm{KO}}$ mutants, we characterized the progress of lung development in three distinct stages, including E12.5 (embryonic stage), E14.5 (pseudoglandular stage), and E16.5 (canalicular stage), by gross morphological examination and histological analysis (Fig. 1B). $\mathrm{Ccrk}^{\mathrm{KO}}$ mutant homozygous lungs showed normal morphology on E12.5, whereas they exhibited retarded lung growth associated with a reduction in regularly shaped developing alveoli in the pseudoglandular stages. In addition, alveoli number and size in $\mathrm{Ccrk}^{\mathrm{KO}}$ mutant embryos were markedly reduced and separated with a thickened interstitium at E14.5. These abnormal formations of the terminal bronchioles indicated defects in lung-

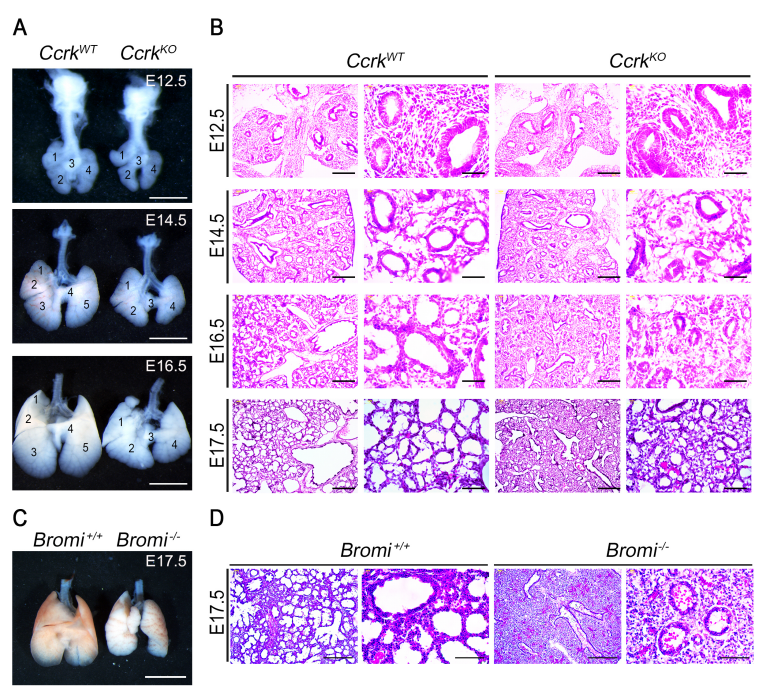

Fig. 1. CCRK is a crucial regulator for proper lung development (A) Whole-mount lungs isolated from E12.5, E14.5, and E16.5 embryos. $C_{c r k}{ }^{K O}$ mutants showed fewer branching lobes and appeared smaller than their wild-type littermates. Scale bars $=1 \mathrm{~mm}$. (B) Histological analysis of embryonic lung sections from indicated embryonic stages. Scale bars $=100 \mu \mathrm{m}$ and $50 \mu \mathrm{m}$. (C) Images from whole-mount lungs isolated from E17.5 wild-type and Bromi $^{-l-}$ mutant embryos. (D) H\&E staining of histological sections shows less saccule formation and thickening of the lung parenchyma in Bromi $^{-1-}$ mutants than in controls. Scale bars $=100 \mu \mathrm{m}$ and 50 $\mu \mathrm{m}$.

branching morphogenesis in $C c r k^{K O}$ mutants. We tested whether the loss of Bromi might phenocopy the hypoplastic lung development of $\mathrm{Ccrk}^{K O}$ mutant embryos. Like the $\mathrm{Ccrk}^{K O}$ mutant respiratory system, $\mathrm{Bromi}^{-1-}$ mutant lungs exhibited fewer lobes, with a significant decrease in lung saccular area and increased mesenchymal thickness (Fig. 1C). This delayed respiratory system development in $\mathrm{CCrk}^{\mathrm{KO}}$ and $\mathrm{Bromi}^{-1-}$ mutants suggests that the CCRK and BROMI complex is required for growth and branching morphogenesis during mouse lung development.

\section{Delayed lung-branching morphogenesis in Ccrk mutant embryos is associated with reduced epithelial-cell proliferation and differentiation}

During lung-branching morphogenesis, various developmental signaling pathways involved in crosstalk between the lung endoderm and surrounding mesoderm determine the branching pattern. To evaluate the effect of Ccrk removal in lung-branching morphogenesis, we immunostained airway endodermal epithelial cells with antibodies for endodermal progenitor regional markers, such as SOX2 and SOX9 for the proximal and distal regions, respectively. Although the overall SOX2 and SOX9 expression patterns in whole-mount lung endoderm were not changed at E12.5, bud growth and branching morphogenesis 
was markedly reduced in the $\mathrm{CCr}^{\mathrm{KO}}$ mutant lung epithelium than in the wild-type control (Fig. 2A). We classified the three different sequential developmental stages of terminal bifurcation as early "bud", "flattened", and later "cleft" stages based on morphology. $\mathrm{C}_{\mathrm{C}} \mathrm{K}^{\mathrm{KO}}$ mutant lungs exhibited relatively increased early bud stages than in the wild-type controls at E14.5 and E16.5 (Fig. 2B and C); however, morphological analysis of later stages of terminal bifurcation of the buds revealed decreased flattened and bifurcated cleft stages of $\mathrm{Ccrk}^{K O}$ mutants. Therefore, $\mathrm{Ccrk}^{\mathrm{KO}}$ mutant lungs predominantly remain in the early stages of bud morphogenesis rather than advancing to the moredeveloped flattened and cleft stages, implying that loss of CCRK leads to delayed bud morphogenesis.

The bronchial tree architecture is formed by extensive proliferation of the distal epithelium and surrounding mesenchyme. We examined coordinated progenitor cell proliferation
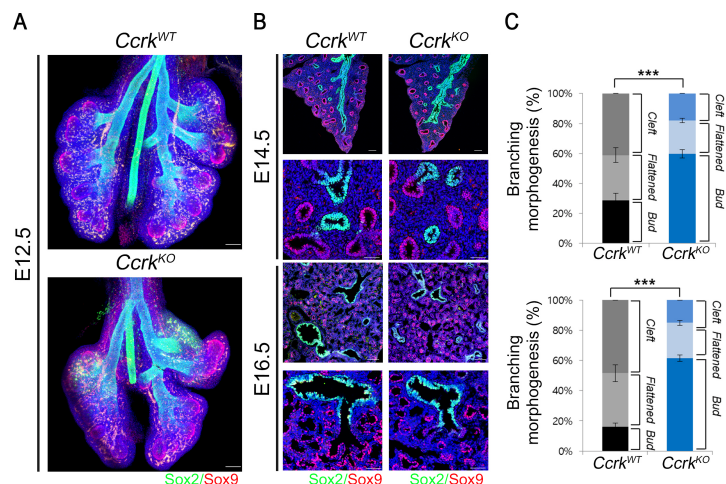

D
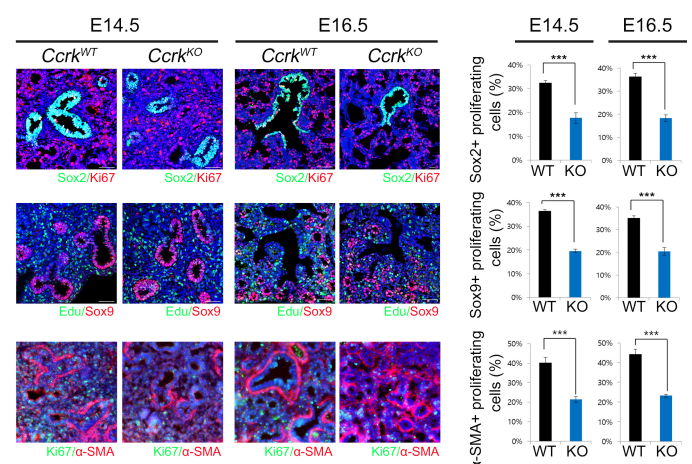

Fig. 2. Ccrk mutant lungs have delayed branching morphogenesis because of decreased epithelial proliferation. (A) Representative whole-mount image of immunofluorescence staining for SOX2 and SOX9 in wild-type and $\mathrm{CCrk}^{K O}$ mutant lungs, which were tissue clearing using clarity. Scale bars $=200 \mu \mathrm{m}$. (B) Sectioned lung tissue were immunostained with SOX2 and SOX9 antibodies. Nuclei were counterstained with DAPI. Scale bars $=100 \mu \mathrm{m}$ and $50 \mu \mathrm{m}$. (C) Statistical analysis of bud morphology during branch bifurcation. (D) We counted $\mathrm{KI} 67$ or EdU immuno-positive proliferating SOX2-, SOX9- or $\alpha$-SMA-positive cells, and obtained the percentage of proliferating cells. Scale bars $=50 \mu \mathrm{m}$. All data are presented as $\mathrm{P}$ values from Student's t-tests: ${ }^{* * *} \mathrm{P}<0.001$ of the lung epithelium and surrounding mesenchyme at E14.5 and E16.5 by immunohistochemistry using the proliferation markers $\mathrm{KI} 67$ or EdU (Fig. 2D and E). We observed that KI67or EdU-labeled proximal or distal epithelial cells in $\mathrm{CCrk}^{\mathrm{KO}}$ mutants were significantly diminished (Fig. 2D and E). Moreover, the proliferation of mesenchymal cells in $\mathrm{Ccrk}^{\mathrm{KO}}$ mutant lungs was also decreased, suggesting that the effects of loss of Ccrk on epithelial and mesenchymal progenitor-cell proliferation may be the primary cause of lung hypoplasia and delayed bud morphology. To further examine the effects of delayed terminal bifurcation and impaired epithelial cell proliferation in $\mathrm{C}_{\mathrm{CK}}{ }^{\mathrm{KO}}$ embryos on SOX2 and SOX9 endoderm progenitor differentiation, we analyzed the expression levels of the endodermal and epithelial lineage cell differentiation markers (Supplementary Fig. 2). All differentiated cell marker expression was significantly diminished in mutant embryos. Therefore, CCRK is important for epithelial progenitor cell proliferation and differentiation in mouse lung development.

\section{CCRK is essential for Hh signaling in lung mesenchymal progenitor cells via regulation of primary cilia formation} The $\mathrm{SHH}$ signaling pathway positively regulates epithelial-cell proliferation by increasing FGF10 expression in mesenchymal cells during the process of lung branching morphogenesis. $\mathrm{SHH}$ expression is restricted to the distal epithelium in developing lungs. Ptch1 and Gli1 are highly expressed in the mesenchyme that surrounds epithelial cells around the branching tips. In addition, bone morphogenic protein 4 (BMP4) is also expressed in the distal epithelium during the pseudoglandular stage and is required for promoting epithelial cell proliferation for branch morphogenesis. To investigate the defect in signaling pathways that regulate epithelial-cell proliferation in $\mathrm{CCrk}^{\mathrm{KO}}$ mutant lungs, we examined the expression of Shh and Bmp4 by RNA in situ hybridization analysis at E14.5. We found that $\mathrm{Ccrk}^{\mathrm{KO}}$ mutants showed significantly downregulated expression of Shh and Bmp4 in the epithelium. Also, the expression of $\mathrm{SHH}$ target genes was also downregulated in $\mathrm{Ccrk}^{K O}$ mutant lungs. These results revealed a significant impairment of $\mathrm{Hh}$ signaling in mesenchymal cells in $\mathrm{CCrk}^{\mathrm{KO}}$ mutant lungs (Fig. 3A). Moreover, BMP4, which is normally modulated by $\mathrm{SHH}$-signal transduction in the distal epithelium, was downregulated in the $\mathrm{Ccrk}^{\mathrm{KO}}$ mutants, suggesting that $\mathrm{SHH}$ signaling is affected by loss of CCRK function. To confirm the compromised $\mathrm{SHH}$ signaling in $C \mathrm{Crk}{ }^{\mathrm{KO}}$ mutants, we examined the gene expression of signaling pathways that regulate branching morphogenesis using quantitative real-time PCR. Consistently, the SHH target genes were downregulated in $\mathrm{C} C \mathrm{Ck}^{\mathrm{KO}}$ mutant lungs. Bmp4 was also significantly downregulated in $\mathrm{C}_{\mathrm{C}} \mathrm{K}^{\mathrm{KO}}$ mutant lungs. In contrast, expression of genes, such as Wht and Fgf10, involved in epithelial and mesenchymal proliferation during branch morphogenesis, were not affected (Fig. 3B). These data suggest that CCRK functions as a positive regulator of $\mathrm{Hh}$ signaling during lung development and that the defects of epithelial and 
A

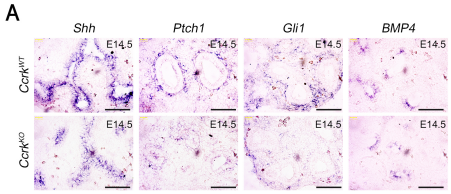

B

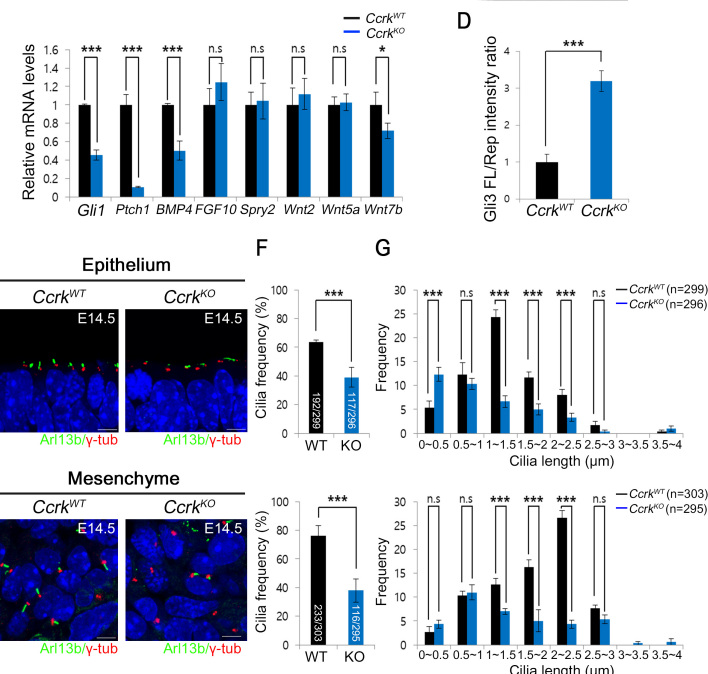

Fig. 3. Loss of Ccrk results in downregulation of the $\mathrm{SHH}$ signaling pathway and ciliary defects in the pseudoglandular stage. (A) In situ hybridization lung-tissue sections displayed expression of Shh, Gli1, Ptch1, and Bmp4. Scale bars $=50 \mu \mathrm{m}$. (B) Target gene expression for SHH, FGF, and Wnt pathways in the developing lungs of wild-type and $\mathrm{Ccrk}^{K O}$ mutant lungs. $\mathrm{P}$ values are from Student's t-tests: $* * * P<0.001$, ${ }^{*} P<0.05$; n.s., not significant. (C) Immunoblots of GLI3 in E14.5 whole-lung tissue extracts. $\beta$-actin served as a loading control. (D) Quantification of GLI3-FL/Rep ratios. Two samples for each genotype were analyzed. $\mathrm{P}$ values are from Student's t-tests: $* * * \mathrm{P}<0.001$. (E) We analyzed primary cilia in the E14.5 $\mathrm{CCrk}^{\mathrm{KO}}$ mutant and wildtype lung epithelium and mesenchyme using confocal microscopy by staining with ARL13B and $\gamma$-TUB antibodies. Scale bars $=5 \mu \mathrm{m}$. (F) We counted the number of primary cilia in each genotype and then divided by the number of total $\gamma$-TUB-positive cells. P values are from Student's t-tests: $* * * P<0.005$. (G) Distribution of primary cilia in $\mathrm{Ccrk}^{K O}$ mutants and wild-type lungs was measured. Average lengths and standard deviations for distributions are shown. $\mathrm{P}$ values are from Student's t-tests: $* * * \mathrm{P}<0.005 ;$ n.s., not significant.

mesenchymal cell proliferation observed in $\mathrm{CCrk}^{\mathrm{KO}}$ mutants may be due, in part, to altered $\mathrm{Hh}$ and BMP4 signaling. Although GLI3 exists in a full-length activator form or a processed repressor form, functioning as both positive and negative regulators of the $\mathrm{SHH}$ signaling pathway, the repressor function has a dominant role in embryonic lung development. We evaluated whether GLI3 processing is also affected in $\mathrm{Ccrk}^{K O}$ mutant lungs. We found that GLI3 processing was decreased in $\mathrm{CCrk}^{\mathrm{KO}}$ mutant lungs, resulting in an increased ratio of full-length GLI3 to GLI3 repressor, thus indicating compromised $\mathrm{Hh}$ signaling (Fig. 3C and D).

Primary cilia are temporally present in progenitor cells and

differentiate to multi-cilia, but other cell types maintain the non-motile primary cilia after cell differentiation. To characterize the morphology and frequency of primary cilia in $\mathrm{Ccrk}^{\mathrm{KO}}$ mutant lungs at the pseudoglandular stage, we examined the primary cilia by immunostaining for the ciliary marker ARL13B and the basal body marker $\gamma$-tubulin ( $\gamma$-TUB) (Fig. 3D). Both epithelial and mesenchymal cells in $\mathrm{CCrk}^{K O}$ mutant embryonic lungs displayed swelling at their distal tips and shortened ciliary length compared with those in wild-type embryonic lungs. We measured the ciliary frequency of wild-type and $\mathrm{Ccrk}^{\mathrm{KO}}$ mutant embryonic lung epithelial and mesenchymal cells (Fig. 3E). $\mathrm{C}_{\mathrm{C}} \mathrm{K}^{\mathrm{KO}}$ showed reduced ciliary frequency in both the epithelium and the mesenchyme in embryonic lungs and the ciliary lengths in $\mathrm{CCrk}^{\mathrm{KO}}$ mutant more broadly distributed than control (Fig. 3F). Therefore, CCRK regulates the proper length of primary cilia in epithelium and mesenchymal progenitor cells to regulate $\mathrm{Hh}$ signaling; these events may be important for development of branching morphogenesis.

Next, to evaluate the motile multi-ciliogenesis of ciliated epithelial cells under conditions of disrupted primary ciliogenesis in $\mathrm{CCrk}^{\mathrm{KO}}$ mutants, we examined the generation of multi-cilia with antibodies to cilia markers ARL13B and acetylated $\alpha$-tubulin (Ac-TUB) as well as the motile cilia marker $\beta$-TUB IV with ARL13B to confirm the ciliary type (Supplementary Fig. 3). Similar to primary ciliogenesis defects in progenitor cells, the removal of the Ccrk gene in differentiated ciliated cells impaired motile multi-cilia formation at the canalicular stage. Moreover, we analyzed the expression profiles of key ciliogenic-transcription factors and common ciliary genes during primary cilia (E14.5) and multi-cilia stages (E17.5) in CCrk ${ }^{K O}$ mutant and control embryos (Supplementary Fig. 4). Interestingly, the Ccrk mutant displays a significantly reduced expression of ciliary genes at E17.5 but not in E14.5. These results indicate that CCRK might involve in two ciliogenesis processes with different mechanisms. Collectively, CCRK is required for proper assembly of ciliogenesis in both primary cilia and multiple motile cilia.

\section{Activation of $\mathrm{Hh}$ signaling partially rescues the branching morphogenesis defect in Ccrk mutant explant lungs}

To examine whether the decreased $\mathrm{Hh}$ signaling pathway in $\mathrm{CCrk}^{\mathrm{KO}}$ mutants is the primary cause of dysregulation in branching morphogenesis in vitro, we manipulated the $\mathrm{Hh}$ signaling pathway in an explant culture system by using small chemical Smoothened agonist SAG. After about $48 \mathrm{~h}$ of SAG treatment, wild-type explants did not exhibit significant alterations in bud-branching morphogenesis (Fig. 4A). The $\mathrm{CCrk}^{\mathrm{KO}}$ mutant lungs cultured in vitro exhibited branching defects similar to those we observed in vivo, displaying a significantly decreased number of branches after $48 \mathrm{~h}$ of culture. However, treatment with 500 nM SAG efficiently increased the growth of bud branching and the number of branches in the mutant lungs, yielding results comparable with the control lungs. Consistently, SAG treatment of wild-type lung explants for $48 \mathrm{~h}$ increased the expression of the $\mathrm{Hh}$ 
A

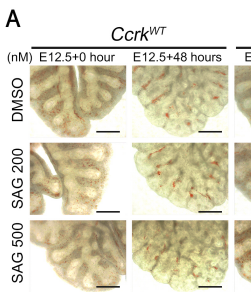

Ccrk ${ }^{\kappa O}$ C

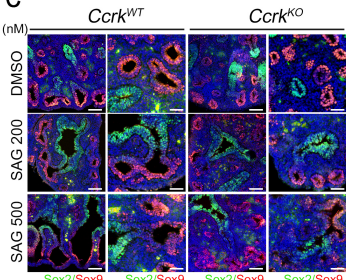
D
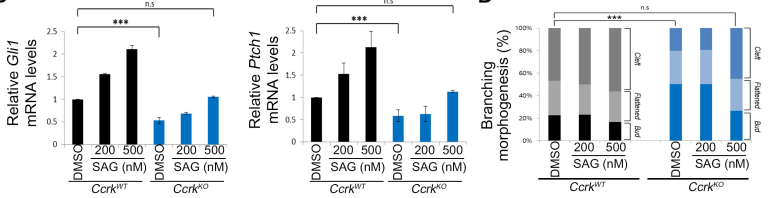
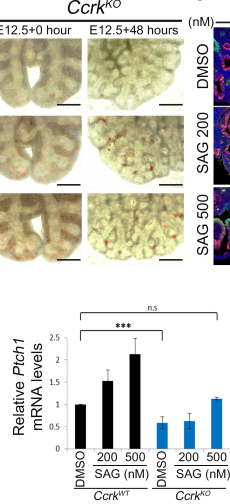

Fig. 4. SAG treatment of $\mathrm{Ccrk}^{K O}$ mutant explant lungs rescues $\mathrm{Hh}$ signaling and lung branching morphogenesis. (A) Embryonic lungs were dissected at E12.5 and treated for $48 \mathrm{~h}$ with or without SAG-enriched media with serum. Scale bars $=100 \mu \mathrm{m}$. (B) The q-PCR analysis of Gli1 and Ptch1 in explant lungs treated with SAG or DMSO. (C) Sectioned lung tissue was stained for SOX2 and SOX9 antibodies to show the development of lungs. Scale bars $=50 \mu \mathrm{m}$. (D) Statistical analysis compared bud morphology during branch bifurcation. All data are presented as $\mathrm{P}$ values from Student's t-tests: $* * * \mathrm{P}<0.001 ;$ n.s., not significant.

target genes Gli1 and Ptch1 in a dose-dependent manner. Although the responsiveness to SAG in $\mathrm{CCrk}^{K O}$ mutants was significantly lessened compared to that of the wild-type controls, it modestly increased Gli1 and Ptch1 expression compared to that of the DMSO control (Fig. 4B). The increased expression levels of Gli1 and Ptch1 in Ccrk ${ }^{K O}$ explant lungs following $500 \mathrm{nM}$ SAG treatment was comparable to that in DMSO-treated wild-type explant lungs. Moreover, E12.5 explant lungs from wild-type and mutant embryos were further treated with DMSO or SAG for $48 \mathrm{~h}$ and sectioned for analysis of terminal bifurcation via detection of SOX2 and SOX9 (Fig. 4C and D). The 500-nM SAG treatment significantly increased the SOX9-positive nascent bud size and number in the distal epithelium of the $C_{c r k}{ }^{K O}$ mutant explant lungs. Although the relative percentage of the cleft form did not change in 200-nM SAG-treated $\mathrm{Ccrk}^{K O}$ mutant explant lungs, 500-nM SAG treatment increased the cleft and flattened forms in mutants at the expense of the bud form. These findings demonstrate that the effective increase of $\mathrm{Hh}$ response in developing lungs of $\mathrm{Ccrk}^{\mathrm{KO}}$ mutants partially recovers the branching morphogenesis, implying that defects in progenitor-cell proliferation and lung-branching morphogenesis may result from the primary decrease in $\mathrm{Hh}$ signaling in $\mathrm{C} C r k^{K O}$ mutants.

\section{DISCUSSION}

CCRK has been identified as a CDK-family kinase and is aberrantly overexpressed in various types of cancers $(14,15)$. Recent mouse studies indicate that Ccrk regulates ciliogenesis and is involved in embryonic organ development via Hh-signal transduction (12). Here, we found that CCRK regulates epithelial and mesenchymal progenitor-cell proliferation, in part, by controlling the ciliogenesis and $\mathrm{Hh}$ signaling during embryonic lung development.

To form a branched conducting and air-blood gas exchange system in respiratory organs, timely interplay between signaling pathways is important during embryonic lung development. Patterning of terminal branching requires autocrine and paracrine signaling via the FGF, BMP, Wnt, and Hh signaling pathways to regulate proper epithelial-cell proliferation, bud branching, and differentiation. Our Ccrk mutant mouse embryos showed hypoplastic lung development and disrupted terminal branching morphogenesis at the pseudoglandular stage. The control of proper patterning of terminal bud branching relies on distal epithelial proliferation after initial bud outgrowth. The developmental genetic programs are hard-wired for lung epithelial progenitor-cell proliferation during the pseudoglandular stage. Signals from FGF10, BMP4, $\mathrm{SHH}$, and WNT ligands with a mesenchymal or epithelial origin increase epithelial cell proliferation. In Ccrk mutant embryonic lungs from the pseudoglandular stage, we observed reduced expression of Bmp4, Shh, and Wnt7b and diminished cell proliferation in the distal region of the lung buds (Figs. 2D, $3 \mathrm{~A}$ and $\mathrm{B})$. During the pseudoglandular stage, epithelial BMP4 expression is restricted to the tip of lung buds; inactivation of BMP4 signaling via lung epithelium-specific deletion of Bmp4 results in decreased epithelial proliferation (16). The underlying molecular mechanisms of reduced epithelial cell proliferation and BMP4 and $\mathrm{SHH}$ expression may result from an intertwined gene-expression program in developing lungs. Mesenchymal FGF10 positively regulates BMP4 expression and is important for maintenance of $\mathrm{SHH}$ expression in the epithelium. The dynamic expression of FGF10 is dependent on epithelial $\mathrm{SHH}$ expression; however, FGF10 expression is not changed in Ccrk mutant embryos. Therefore, low-level expression of BMP4 and SHH may be caused by diminished activity of FGF10 rather than by its expression in the distal epithelial compartment. Interestingly, our previous study of cultured cells indicated that FGF receptor signaling may be linked to CCRK and other kinases (17).

Our previous and current studies show that CCRK functions as a key regulator of ciliogenesis and Hh signaling. CCRK regulates another ciliary length-controlling kinase, intestinal cell kinase (ICK), in mammals via phosphorylation of a key catalytic active motif, the TDY domain. The human congenital disorder endocrine-cerebro-osteodysplasia (ECO) syndrome is caused by a homozygous loss-of-function mutation in ICK. Recently, a knock-in mouse model for ECO mutation was generated (18). Interestingly, the homozygous Ick mutant lung displays impaired lung-branching morphogenesis and abnormal ciliogenesis similar to that of the Ccrk mutant. In our studies, Ccrk mutant mouse lungs exhibited defects in primary cilia formation in epithelial and mesenchymal progenitor cells and 
impaired Hh signaling (Fig. 3A-D). It is possible that aberrant signaling crosstalk during branching morphogenesis due, in part, to failure to form primary cilia results in diminished responsiveness to $\mathrm{Hh}$ signaling in the mesenchymal compartment. Moreover, activation of Hh signaling via SAG treatment in lung explant cultures was sufficient to rescue the lungbranching morphogenesis defects observed in Ccrk mutants.

Our data provide evidence underscoring the importance of ciliogenesis and $\mathrm{SHH}$ signaling in lung development and disease $(5,19)$. There are significant phenotypic similarities in lung development between the Ccrk mutant and Gli mutant (Gli2 and Gli3) mouse embryos, which are key components in the Hh signaling pathway. Both Gli2 and Gli3 mutant embryos display respiratory failure linked to the absence of functional alveoli with hypoplasia $(7,8)$. In both Gli2 and Gli3 mutant lungs, decreased epithelial-cell proliferation may contribute to the hypoplastic lungs that typically are decreased in size and have severe patterning defects.

In this study, we found that loss of Ccrk can lead to pulmonary hypoplasia with abnormal branching morphogenesis and severe alveolar airspace deficiency during embryonic development. Epithelial cell proliferation during the pseudoglandular stage and interstitial epithelial-cell differentiation and remodeling during lung sacculation are important cellular mechanisms by which CCRK regulates development of embryonic respiratory organs. Loss of Ccrk function perturbed not only ciliary structure and function but also Hh signaling, a highly conserved intracellular process involved in the maintenance of cell homeostasis. Further studies to elucidate the precise mechanisms by which CCRK and primary cilia exert their effects on the complicated signaling pathways involved in lung development and branching morphogenesis during early embryonic stages are warranted.

\section{ACKNOWLEDGEMENTS}

This research was supported by the Basic Science Research Program (NRF-2015M3A9B6027820, NRF-2018M3C7A1057313 to H.W.K.), and Korea Mouse Phenotyping Project (NRF2014M3A9D5A01073969) funded through the NRF by the Korean government (MSIP).

\section{CONFLICTS OF INTEREST}

The authors have no conflicting interests.

\section{REFERENCES}

1. Morrisey EE and Hogan BL (2010) Preparing for the first breath: genetic and cellular mechanisms in lung development. Dev Cell 18, 8-23

2. Herriges M and Morrisey EE (2014) Lung development: orchestrating the generation and regeneration of a complex organ. Development 141, 502-513

3. Warburton D, Bellusci S, De Langhe S et al (2005) Molecular mechanisms of early lung specification and branching morphogenesis. Pediatr Res 57, 26R-37R

4. Ingham PW and McMahon AP (2001) Hedgehog signaling in animal development: paradigms and principles. Genes Dev 15, 3059-3087

5. Kugler MC, Joyner AL, Loomis CA and Munger JS (2015) Sonic hedgehog signaling in the lung. From development to disease. Am J Respir Cell Mol Biol 52, 1-13

6. Pepicelli CV, Lewis PM and McMahon AP (1998) Sonic hedgehog regulates branching morphogenesis in the mammalian lung. Curr Biol 8, 1083-1086

7. Li Y, Zhang H, Choi SC, Litingtung Y and Chiang C (2004) Sonic hedgehog signaling regulates $\mathrm{Gli3}$ processing, mesenchymal proliferation, and differentiation during mouse lung organogenesis. Dev Biol 270, 214-231

8. Rutter M, Wang J, Huang Z, Kuliszewski $M$ and Post $M$ (2010) Gli2 influences proliferation in the developing lung through regulation of cyclin expression. Am J Respir Cell Mol Biol 42, 615-625

9. Ko HW (2012) The primary cilium as a multiple cellular signaling scaffold in development and disease. BMB Rep 45, 427-432

10. Reiter JF and Leroux MR (2017) Genes and molecular pathways underpinning ciliopathies. Nat Rev Mol Cell Biol $18,533-547$

11. Jain R, Pan J, Driscoll JA et al (2010) Temporal relationship between primary and motile ciliogenesis in airway epithelial cells. Am J Respir Cell Mol Biol 43, 731-739

12. Snouffer A, Brown D, Lee H et al (2017) Cell Cycle-Related Kinase (CCRK) regulates ciliogenesis and Hedgehog signaling in mice. PLoS Genet 13, e1006912

13. Ko HW, Norman RX, Tran J, Fuller KP, Fukuda $M$ and Eggenschwiler JT (2010) Broad-minded links cell cyclerelated kinase to cilia assembly and hedgehog signal transduction. Dev Cell 18, 237-247

14. Wang $\mathrm{R}, \mathrm{Xu} \mathrm{XY}, \mathrm{Zhu} \mathrm{H}$ et al (2019) Androgen Receptor Promotes Gastric Carcinogenesis via Upregulating Cell Cycle-Related Kinase Expression. J Cancer 10, 4178-4188

15. Yang Y, Roine N and Makela TP (2013) CCRK depletion inhibits glioblastoma cell proliferation in a cilium-dependent manner. EMBO Rep 14, 741-747

16. Weaver M, Yingling JM, Dunn NR, Bellusci $S$ and Hogan BL (1999) Bmp signaling regulates proximal-distal differentiation of endoderm in mouse lung development. Development $126,4005-4015$

17. Kunova Bosakova M, Nita A, Gregor T et al (2019) Fibroblast growth factor receptor influences primary cilium length through an interaction with intestinal cell kinase. Proc Natl Acad Sci U S A 116, 4316-4325

18. Moon H, Song J, Shin JO et al (2014) Intestinal cell kinase, a protein associated with endocrine-cerebro-osteodysplasia syndrome, is a key regulator of cilia length and Hedgehog signaling. Proc Natl Acad Sci U S A 111, 8541-8546

19. Fernandes-Silva H, Correia-Pinto J and Moura RS (2017) Canonical Sonic Hedgehog Signaling in Early Lung Development. J Dev Biol 5, 3 